\title{
Ruptured thymoma causing a hemothorax: A case report
}

\author{
DAISUKE HOKKA, HIROYUKI OGAWA, SHINYA TANE, YUGO TANAKA, \\ SHUNSUKE TAUCHI and YOSHIMASA MANIWA
}

\begin{abstract}
Division of Thoracic Surgery, Department of Surgery, Kobe University Graduate School of Medicine, Kobe, Hyōgo 650-0017, Japan
\end{abstract}

Received July 17, 2014; Accepted April 8, 2015

DOI: $10.3892 / \mathrm{ol} .2015 .3476$

\begin{abstract}
A thymoma is a neoplasm that arises from the epithelial cells of the thymus, and may cause various signs and symptoms dependent upon its local extent. A non-traumatic hemothorax is extremely rare. The present study reports the case of a 77-year-old female who presented with an acute onset of chest pain. Imaging procedures revealed a mass occupying the anterior mediastinum and left hemithorax, and a left pleural effusion. Progressive anemia was noted following admission. Left hemothorax due to rupture of the anterior mediastinal mass was suspected, and emergency surgery was performed. Hemorrhage was observed on the cut surface of the tumor. An analysis of frozen sections indicated a thymoma, and a thymo-partial thymectomy was subsequently performed to remove as much of the hematoma as possible. The patient was discharged on post-operative day 13 following an uneventful recovery. The present case suggests that in previously healthy individuals, sudden-onset dyspnea and chest pain co-occurring with an acute widening of the mediastinum observed on roentgenograph may be indicative of a ruptured thymoma.
\end{abstract}

\section{Introduction}

Thymomas are unique neoplasms that originate in the epithelial tissue of the thymus (1). The prevalence of thymomas is equal in males and females, and the age at diagnosis is typically 30-40 years, however, cases have been described in all age groups, including rare occurrences in children (1). In adults, thymoma accounts for $20 \%$ of anterior mediastinal neoplasms, and it is thus the most common neoplasm of the anterior mediastinum (2). Thymomas are typically detected during routine radiographs and in myasthenia gravis patients, $\sim 15 \%$ of whom are affected $(1,2)$. These tumors are commonly

Correspondence to: Dr Daisuke Hokka, Division of Thoracic Surgery, Department of Surgery, Kobe University Graduate School of Medicine, 7-5-2 Kusunoki, Chūō-ku, Kobe, Hyōgo 650-0017, Japan E-mail: daigoro1019@yahoo.co.jp

Key words: ruptured thymoma, chest pain, urgent surgery, hemothorax associated with indolent growth and with a variety of paraneoplastic syndromes, most frequently myasthenia gravis (3). Approximately one-third of thymoma patients exhibit superior vena cava syndrome, coughing, chest pain or dysphagia; these symptoms result from compression of the surrounding organs by an expansive mass (1). Surgery is the predominant treatment method for thymoma. However, if there is indication that the tumor is large and invasive, pre-operative neoadjuvant chemotherapy and/or radiotherapy may be administered in order to reduce the size of the mass and to improve resectability prior to surgery (1). Stage III and IV thymomas have a markedly poorer prognosis compared with stage I and II tumors. Metastasis of invasive thymomas may also occur, however, this is uncommon. Sites of metastasis generally include the pleura, bones, liver or brain, occurring in $\sim 7 \%$ of cases (1).

Non-traumatic hemothorax is a rare condition, which generally occurs due to the erosion of a vessel caused by a pathological lesion. In tuberculosis of the lung, the pulmonary arteries or their branches are the vessels generally involved (4). The present study reports a rare case of hemothorax caused by a ruptured thymoma, which necessitated urgent surgery.

\section{Case report}

A 77-year-old, previously healthy female was referred to Kobe University Hospital (Kobe, Hyōgo, Japan) hospital with an acute onset of chest pain in December, 2012. Chest roentgenography and computed tomography revealed a mass occupying the anterior mediastinum and left hemithorax, a left-sided pleural effusion, and a normal aorta and great vessels (Fig. 1). Progressive anemia was noted following admission. A diagnosis of a left hemothorax caused by rupture of the anterior mediastinal mass was suspected, and emergency surgery was conducted. Upon opening the left pleural cavity, $\sim 1,350 \mathrm{ml}$ of blood was released. An anterolateral thoracotomy was performed by video-assisted thoracoscopic surgery, revealing a large, encysted hematoma along the tumor $(72 \times 43 \times 43 \mathrm{~mm})$, extending from the left anterior mediastinum into the left pleural cavity and ending with a rupture. Hemorrhage on the cut surface of the tumor was observed (Fig. 2A and B). An analysis of frozen sections using hematoxylin and eosin staining (Sakura Finetek Nihonbashi-Hamacho, Tokyo, Japan) revealed a hemorrhagic lesion with lymphocytic infiltration and 

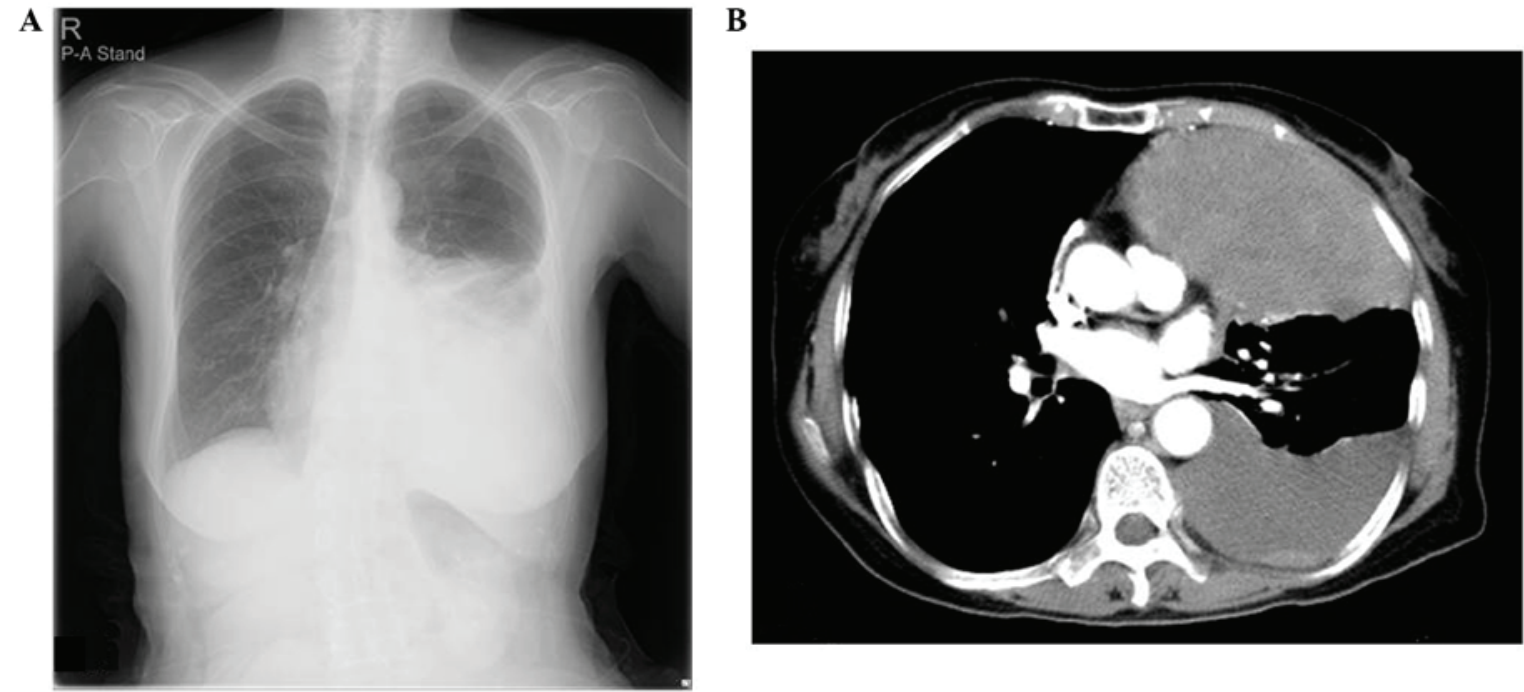

Figure 1. (A) Chest roentgenography and (B) computed tomography scans showing a mass occupying the anterior mediastinum and left hemithorax, a left pleural effusion, and a normal aorta and great vessels.
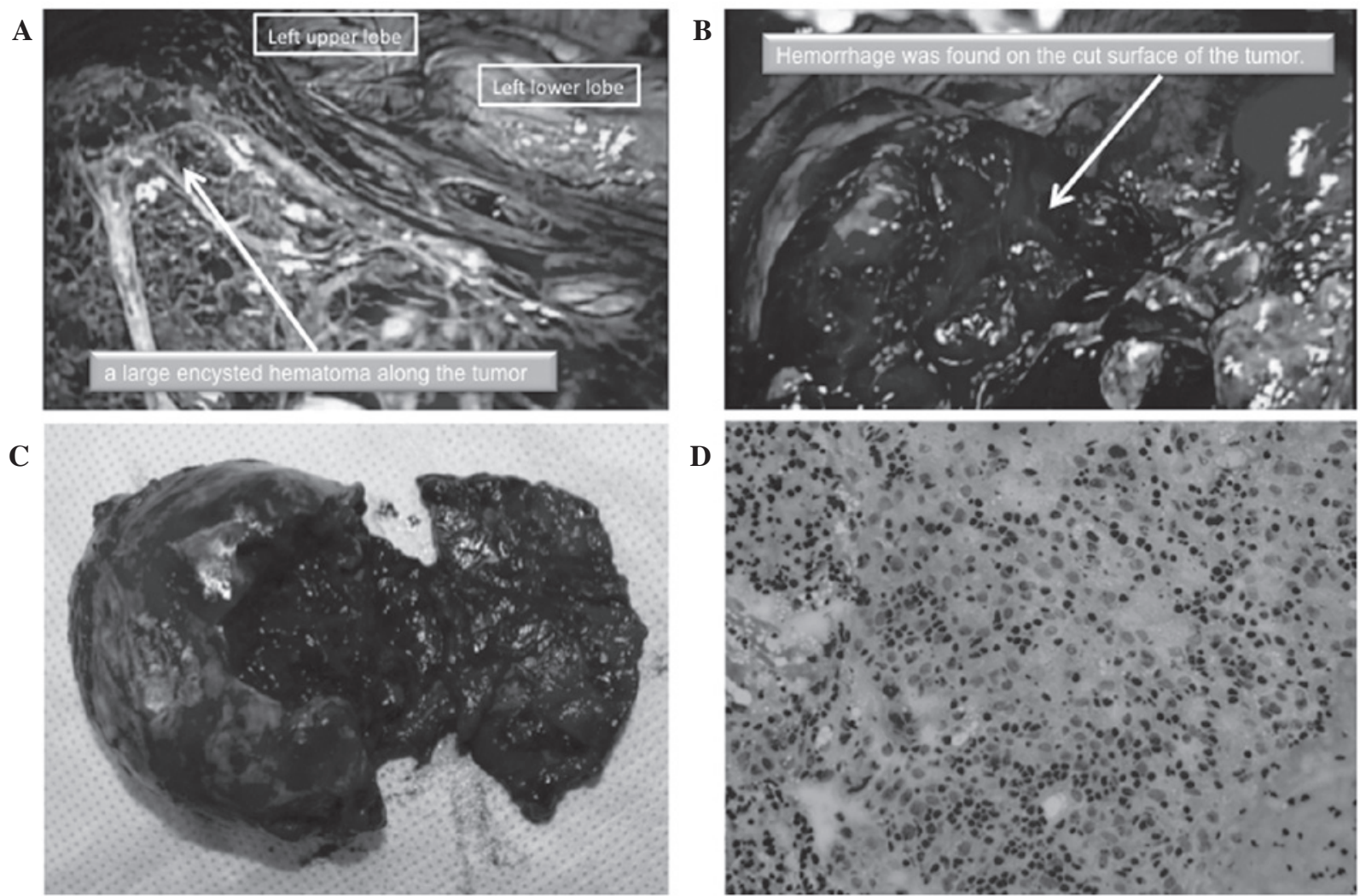

D

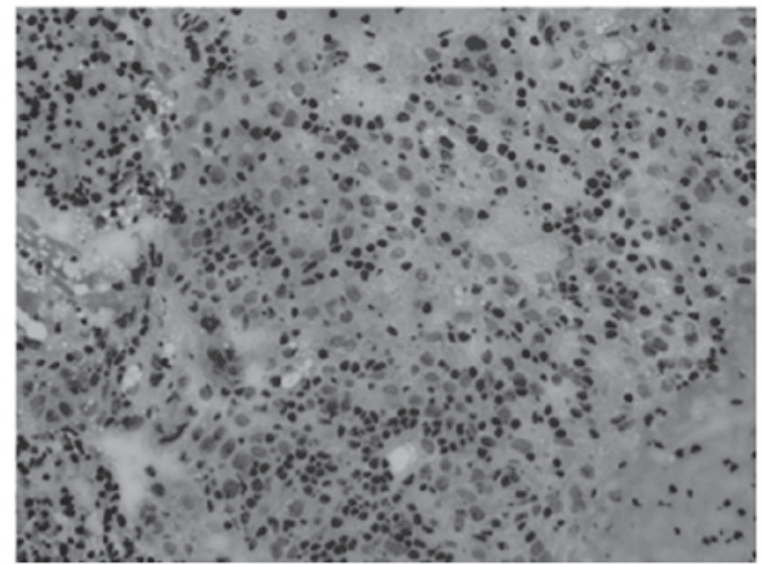

Figure 2. (A) Anterolateral thoracotomy revealed a large encysted hematoma along the tumor, extending from the left anterior mediastinum into the left pleural cavity and ending with a rupture. (B) Hemorrhage was found on the cut surface of the tumor. (C) The thymoma was completely resected en bloc with part of the left upper lobe. (D) Pathological examination using hematoxylin and eosin staining (magnification, x200) demonstrated a lymphocyte-rich tumor, leading to the diagnosis of type B1 thymoma (World Health Organization classification) with no capsular invasion. The tissue was characterized by broad fibrous bands separating proliferating epithelial cells admixed with small lymphoid cells. The epithelial component was highlighted by a cytokeratin AE1/AE3 immunostain. LUL, left upper lobe; LLL, left lower lobe.

epithelial elements, indicative of thymoma, and a thymo-partial thymectomy was subsequently performed, removing the maximal possible volume of hematoma. The thymoma was completely resected en bloc with part of the left upper lobe of the thymus (Fig. 2C). A pathological examination revealed that the tissue was composed of broad fibrous bands separating proliferating epithelial cells (highlighted by cytokeratin AE1/ AE3 immunostaining) admixed with small lymphoid cells, indicating a diagnosis of lymphocyte-rich (type B1) thymoma (World Health Organization classification) $(5,6)$ (Fig. 2D). The 
tumor showed hemorrhage and rupture, and no capsular invasion (Masaoka stage 2), and was continuous with the hematoma resulting from partial destruction of its epithelial lining (1). In addition, coagula and fibrin calculi contained tissue from the thymoma, and a diagnosis of hemothorax formation due to hemorrhage from the ruptured thymoma was determined. The patient was discharged on post-operative day 13 following an uneventful recovery, and was in good health at the six-month follow-up examination.

Written informed consent for the present study was obtained from the patient.

\section{Discussion}

The rupture of mediastinal teratomas has been recognized as a cause of chest pain and massive pleural effusion $(7,8)$; however, the rupture of thymomas is rare. To the best of our knowledge, only six cases, including the present case, of ruptured thymic tumor have been reported. Caplin et al (9) reported a case of a hemothorax resulting from the spontaneous rupture of a thymoma in a 51-year-old male who underwent treatment by posterolateral thoracotomy. Templeton et al (10) reported a case of hemothorax due to rupture of a thymoma simulating aortic dissection in a 63-year-old male; only a biopsy was performed in this case. Fukuse et al (11) reported a case of mediastinal hematoma due to thymomal hemorrhage in a 70-year-old male who was treated by full median sternotomy with anterolateral thoracotomy. Shimokawa et al (12) reported a case of mediastinal hemorrhage and hemothorax due to rupture of a thymoma in a 71-year-old female who subsequently underwent partial sternotomy with hemitransection of the sternum to the left fourth intercostal space. Santoprete et al (13) reported a case of mediastinal hemorrhage and hemothorax due to rupture of a thymoma in a 73-year-old female who underwent treatment by clamshell incision. In these six cases, including the present case, sudden onset of dyspnea and chest pain were the first clinical manifestations and all patients were healthy prior to onset.

In the present case, controlling the left pulmonary hilar vessels and evacuating the hematoma were priorities. Anterolateral thoracotomy with video-assisted thoracoscopic surgery enabled access to the mediastinum and pleural cavities, and rapid control of the tumor. The present case, as well as other previously reported cases, illustrates that sudden-onset dyspnea and chest pain in a previously healthy individual, which co-occurs with acute mediastinal widening on chest roentgenography, may be indicative of ruptured thymoma.

\section{References}

1. Thomas CR, Wright CD and Loehrer PJ: Thymoma: State of the art. J Clin Oncol 17: 2280-2289, 1999.

2. Gerein AN, Srivastava SP and Burgess J: Thymoma: A ten year review. Am J Surg 136: 49-53, 1978.

3. Loehrer PJ Sr, Jiroutek M, Aisner S, Aisner J, Green M Thomas CR Jr, Livingston R and Johnson DH: Combined etoposide, ifosfamide, and cisplatin in the treatment of patients with advanced thymoma and thymic carcinoma: An intergroup trial. Cancer 91: 2010-2015, 2001.

4. Janik M, Straka L, Krajcovic J, et al: Non-traumatic and spontaneous hemothorax in the setting of forensic medical examination: A systematic literature survey. Forensic Sci Int 236: 22-29, 2014.

5. Rosai J and Sobin LH (eds): World Health Organization. International Histological Classification of Tumours: Histological Typing of Tumours of the Thymus. 2nd edition. Springer-Verlag Berlin Heidelberg, Germany, 1999.

6. Okumura M, Ohta M, Tateyama $\mathrm{H}$, et al: The World Health Organization histologic classification system reflects the oncologic behavior of thymoma: A clinical study of 273 patients. Cancer 94: 624-632, 2002.

7. Choi SJ, Lee JS, Song KS and Lim TH: Mediastinal teratoma: CT differentiation of ruptured and unruptured tumors. AJR Am J Roentogenol 171: 591-594, 1998.

8. Sasaka K, Kurihara Y, Nakajima Y, et al: Spontaneous rupture: A complication of benign mature teratomas of the mediastinum. AJR Am J Roentogenol 170: 323-328, 1998.

9. Caplin JL, Gullan RW, Dymond DS, Bradley SMO, Hill IM and Banim SO: Hemothorax due to rupture of a benign thymoma. Jpn Heart J 26: 123-125, 1985.

10. Templeton PA, Vainright JR, Rodriguez A and Diaconis JN: Mediastinal tumors presenting as spontaneous hemothorax, simulating aortic dissection. Chest 93: 828-830, 1988.

11. Fukuse T, Matsukura T, Nakamura A, Kosaka S and Tamada J: Mediastinal hematoma due to thymoma hemorrhage - A case report. Nihon Kyobu Geka Gakkai Zasshi 39: 930-934, 1991 (In Japanese).

12. Shimokawa S, Watanabe S, Sakasegawa K and Tani A: Ruptured thymoma causing mediastinal hemorrhage resected via partial sternotomy. Ann Thorac Surg 71: 370-372, 2001.

13. Santoprete S, Ragusa M, Urbani M and Puma F: Shock induced by spontaneous rupture of a giant thymoma. Ann Thorac Surg 83: $1526-1528,2007$. 